\title{
Search and Studies of the First Byurakan Survey Blue Stellar Objects
}

\author{
A. M. Mickaelian *, G. A. Mikayelyan, H. V. Abrahamyan, and G. M. Paronyan \\ NAS RA V. Ambartsumian Byurakan Astrophysical Observatory (BAO), Byurakan 0213, Aragatzotn province, Armenia
}

\begin{abstract}
The First Byurakan Survey (FBS) $2^{\text {nd }}$ Part was devoted to search and studies of Blue Stellar Objects (BSOs) and Late-type Stars. Eleven lists of 1103 BSOs were published in Astrophysics in 1990-1996, found in FBS low-dispersion spectroscopic plates. The selection was carried out in the region with $+33^{\circ}>\delta>$ $+45^{\circ}$ and $\delta>+61^{\circ}$ with a surface of $\sim 4000 \mathrm{deg}^{2}$. As a result, the catalogue of the FBS BSOs was compiled. Its preliminary version has been available at CDS since 1999. We revised and updated the FBS BSOs catalogue with the new data from recently published optical and multiwavelength catalogues to give access to all available data and make further comparative studies of the properties of these objects possible. We made cross-correlations of the FBS BSOs catalogue with the MAPS, USNO-B1.0, SDSS, and 2MASS, as well as ROSAT, IRAS, NVSS, and FIRST catalogs, added updated SIMBAD and NED data for the objects, and provided accurate DSS1 and DSS2 positions and revised photometry. We also checked the objects for proper motion and variability. A refined classification for the low-dispersion spectra in the Digitized First Byurakan Survey (DFBS) was carried out. The revised and updated catalogue of FBS blue stellar objects contains 1101 objects. The FBS blue stellar objects catalogue can be used to study a complete sample of white dwarfs, hot subdwarfs, HBB stars, cataclysmic variables, bright AGN, and to investigate individual interesting objects.
\end{abstract}

Keywords: Astronomical Data Bases: catalogs, Astronomical Data Bases: surveys, stars: subdwarfs, stars: white dwarfs, stars: cataclysmic variables, galaxies: quasars: general, galaxies: Seyfert

\section{Introduction: First Byurakan Survey (FBS)}

The First Byurakan Survey (FBS), also known as Markarian survey, was the first systematic objective prism survey of the extragalactic sky. It was conducted by B. E. Markarian, V. A. Lipovetski and J. A. Stepanian in 1965-1980 at the Byurakan Astrophysical Observatory with the 1m (40") Schmidt telescope and $1.5^{\circ}$ prism (Markarian, 1967, Markarian et al., 1989). At present, the FBS is the largest area spectral survey, covering $17,000 \mathrm{deg}^{2}$ of all the northern sky and part of the southern sky at high galactic latitudes, defined by $\delta>-15^{\circ}$ and $\longrightarrow \mathrm{b} \longrightarrow 15^{\circ}$. The limiting magnitude is $17.5^{m}-18^{m}$, and the dispersion is 1800 $\AA / \mathrm{mm}$ near $\mathrm{H} \gamma$. There are $\sim 40,000,000$ spectra in all FBS plates corresponding to $\sim 20,000,000$ objects; thus, each object has in average two spectra (Markarian et al., 1989). The FBS was conducted originally to search for galaxies with UV-excess (UVX). 1515 UVX galaxies have been discovered (Markarian et al., 1989, 1997, Mazzarella \& Balzano, 1986). Studies of the Markarian galaxies early in the survey led to the spectral classification of Seyfert Galaxies (Weedman \& Khachikyan, 1968), and to the first definition of starburst galaxies (Weedman, 1977).

The huge amount of spectral information contained in the plates allowed the development of several other projects based on the FBS, the most important being the discovery and investigation of blue stellar objects (Abrahamian \& Mickaelian, 1996, Mickaelian, 2000). The FBS led to discovery of various important objects, like Markarian galaxies, FBS blue stellar objects (BSOs), late-type stars, identification of IRAS sources.

The nature of many FBS BSOs is still not clear. Moreover, the lists were published in Astrofizika and were not available for many years electronically. In 1999, the FBS catalog appeared in Vizier (Abrahamian et al., 1999), however with a number of uncertainties and with the old data given in the published papers only. Later in 2004, the accurate positions were measured for all FBS BSOs from two different epochs, DSS1

*aregmick@yahoo.com 
and DSS2 images (Mickaelian, 2004). This revealed a number of proper motion objects, as well as variables and extended objects were revealed. After all these studies, a complete up-to-date catalog of the FBS BSOs was published in 2008 (Mickaelian, 2008), which combined the accurate positions, a reliable photometry, multiwavelength (X-ray, IR, and radio) data, and spectroscopic identifications.

At present the FBS plates have been digitized and the Digitized First Byurakan Survey (DFBS) has been created (Mickaelian et al., 2007). Its images and spectra are available on the DFBS web portal at Astronomical Observatory of Trieste at http://www.ia2-byurakan.oats.inaf.it/ and were used for the present study as well.

\section{The FBS blue stellar objects}

The second part of the FBS was devoted to the discovery and study of blue (UVX) stellar objects (Abrahamian \& Mickaelian 1996 and references therein). It was carried out in 278 fields, in a $4009 \mathrm{deg}^{2} \mathrm{FBS}$ area defined by $+33^{\circ}<\delta<+45^{\circ}$ and $\delta>+61^{\circ}$ ). The discovery of new bright QSOs, Seyferts, planetary nebula nuclei (PNN), cataclysmic variables (CV), white dwarfs (WD), subdwarfs (sd), HBB (Horizontal Branch B) stars, and other interesting objects was the main purpose of this work. It was similar to the Palomar-Green (PG) survey (Green et al. 1986) but used a spectroscopic method of selection (which is more efficient), had a deeper magnitude limit, and larger area (though the Second part did not yet cover the whole FBS area so far). 1103 blue stellar objects (BSOs) have been selected, including 716 new ones. They have been published in 11 lists (Abrahamian \& Mickaelian, 1993a,b, 1994a,b,c, 1995, 1996, Abrahamian et al., 1990a,b,c, 1991). The preliminary catalog of the FBS BSOs is available at the CDS (Abrahamian et al., 1999). And the revised and updated catalogue was published in 2008 (Mickaelian, 2008).

Spectroscopic observations proved the Second part of the FBS to be a rich source of QSOs, Seyferts, WDs, CVs, etc. (Mickaelian et al., 1999, 2001, 2002a, Sinamyan \& Mickaelian, 2006, 2008a,b, 2009). Altogether, spectroscopic observations have been carried out for 406 FBS objects. Based on new spectroscopic observations, the local density of the bright QSOs and the completeness of the Bright Quasar Survey (BQS) (Schmidt \& Green, 1983) have been re-estimated. We now have obtained a surface density of QSOs brighter than $\mathrm{B}=16.16$ (completeness limit of the BQS) in a subarea of the FBS covering $\sim 2,250 \mathrm{deg}^{2}$, equal to 0.012 $d e g^{-2}$, implying a completeness of $53 \pm 10 \%$ (Mickaelian et al., 1999, 2001).

Using the FBS low-dispersion spectra, a rare bright (V 12.6) SW Sex subtype nova-like CV was discovered with a spectroscopic period within the period "gap" for such objects (Mickaelian et al., 2002b).

For a review of the FBS blue stellar objects see Mickaelian (2000) and an analysis of the samples of WDs and CVs see Mickaelian (2005). A full analysis of the FBS sample was given in Sinamyan \& Mickaelian (2011).

The positions for FBS blue stellar objects (BSOs) published in the original papers were rather poor. This made finding the necessary objects very difficult. Later, accurate positions were measured and published for all 1101 FBS BSOs with an accuracy of 1" and better (Mickaelian, 2004). Moreover, both DSS1 and DSS2 (red and blue) positions were measured and the DSS1 and DSS2 positional accuracy was estimated in this paper.

However, the optical magnitudes for the FBS BSOs until recently were rather inaccurate, as well as the multiwavelength data were absent. The Sloan Digital Sky Survey (SDSS, http://www.sdss.org) now gives new photometric and redshift data for many FBS BSOs, which is also very useful for their further studies.

\section{New data for the FBS objects}

Accurate optical positions of the FBS BSOs made possible correct cross-correlation of the sample with all available modern catalogs and databases, like MAPS (Cabanela et al., 2003) and USNO-B1.0 (Monet et al., 2003), SDSS DR6 (Ahumada et al. 2020, online available at http://www.sdss.org/) and 2MASS (Cutri et al. 2003), ROSAT BSC (Voges et al., 1999) and FSC (Voges et al., 2000), IRAS PSC (IRAS, 1988) and FSC (Moshir \& et al., 1990), NVSS (Condon et al., 1998) and FIRST (Becker et al., 2003) radio catalogs. We have also checked our objects in the catalogs of AGN (Véron-Cetty \& Véron, 2006), WDs (McCook \& Sion, 2006), CVs (Ritter \& Kolb, 2003), and the catalogs of SDSS spectroscopically confirmed white dwarfs (Eisenstein et al., 2006), SDSS spectroscopically selected close binary systems (Silvestri et al., 2006), and the lists of SDSS cataclysmic variables (Szkody et al. 2007 and references therein). All these cross-correlations were necessary as not all data are in SIMBAD and NED, as found from other catalogs. 
The identifications from various catalogs (QSO, WD, sd, CV, PN) also allowed to clarify the nature of many FBS objects. In addition, a number of FBS objects (mostly candidate QSOs) have been observed in SDSS and the spectra are available, however not all are classified. We have carried out the classification of these spectra and included the corresponding types in the FBS catalog too. We have also checked the objects for proper motions (PM) and variability, refined the classification using the Digitized First Byurakan Survey (DFBS, Mickaelian et al. 2007) spectra, updated the available data from SIMBAD and NED, and finally prepared a new catalogue of FBS blue stellar objects.

We have also corrected the names for three objects erroneously given in the previous version: FBS 0133+436 instead of FBS 0133+446, FBS 0223+365 instead of FBS 0223+355, and FBS 1600+382 instead of FBS $1609+382$.

\section{Accurate optical positions}

For the new version of the catalogue, we give the accurate DSS1 (McGlynn \& Scollick, 1994) and DSS2 (Lasker et al., 1996) red optical positions using our measurements (Mickaelian, 2004). Both measuremnets were needed to find the proper motions for many objects by comparison of positions from two epochs separated by about 40 years. These accurate positions, as already said and later shown, provided the possibility of correctly finding the corresponding associations in other known catalogues.

\section{Proper motions}

Proper motions (PM) are given in USNO-B1.0, calculated by measurements between POSS I and POSS II observations. However, based on our previous work (Mickaelian, 2004, Mickaelian \& Gigoyan, 2006), when a number of PM from the USNO were not confirmed, we decided to cross-check them independently with our measurements from DSS1 and DSS2. We took the result as a real PM, if both data given in the USNO (in RA and DEC) matched. According to the USNO catalogue, 89 objects in our list have PM>60 mas/yr, including 52 with PM>100 mas/yr. However, by checking the DSS images, 21 objects were rejected. On the other hand, according to our measurements, more 11 objects have large PM not given in USNO catalogue. For objects having PM both by our measurements and USNO data, the agreement is within $30 \%$ (rms is 47 mas/yr and the systematic shift is only 7 mas/yr), which is rather reliable. This means that the USNO PM errors come from incorrect automatic identification of the object in plates of the two epochs, which happens especailly for large PM stars.

To increase the accuracy of the PM given in the catalogue, we have averaged the USNO and our measurements when available.

\section{MAPS/USNO-B1.0 photometry}

To have the best available photometric data for all our objects, we cross-correlated the FBS BSOs list with the Minnesota Automated Plate Scanner (MAPS) catalogue (Cabanela et al., 2003), and the USNOB1.0 (Monet et al., 2003). MAPS is based on measurements from the POSS1, while USNO-B1.0 gives data from both POSS1 and POSS2. We carried out a statistical analysis of the photometric data provided by these two catalogues. When comparing their data from the POSS1 O (B) and E (R) images, we found a systematic shift. For the analysis, we used all FBS objects having USNO-B1.0 B1/B2/R1/R2 data and objects having MAPS (O and E) data.

First, we calculated the systematic shift between the MAPS (O and E) and USNO-B1.0 (B1 and R1) magnitudes. It is 0.01 and -0.05 , respectively, which is negligible. So, we could trust these magnitudes and calculate the more accurate averages both for $\mathrm{O}(\mathrm{B})$ and $\mathrm{E}(\mathrm{R})$. Then we calculated the systematic shift between USNO B1 and B2, and between R1 and R2. It is 0.17 and 0.14 , respectively, which we used for correction of the B2 and R2 magnitudes (as it was rather unlikely that B1 and R1 could have a systematic shift exactly similar to MAPS O and E). The corrected B2 and R2 were used together with MAPS O and $\mathrm{E}$ and USNO B1 and R1 to calculate the average B and R magnitudes, which we believe are the best ones available. Though both MAPS and USNO catalogs give 0.2 as their photometric errors, we find that the rms is about 0.48 for $\mathrm{B}$ magnitudes and 0.34 for $\mathrm{R}$ magnitudes, respectively. 
Note that for all measurements we had eliminated possible variables or accidental large errors. Our conclusion is that $\mathrm{R}$ magnitudes are more accurate and that one should be careful with $\mathrm{B}$ magnitudes, and anyway a correction for the systematic shift is needed to calibrate them.

The rms between MAPS O and USNO B1 is 0.43, between MAPS E and USNO R1 0.50, which is very close to our estimates made for the BIS (Byurakan-IRAS Stars) catalog objects (Mickaelian \& Gigoyan, 2006).

To reach the maximum possible photometric accuracy, we use the average between the MAPS O and USNO B1 as a B magnitude, and when MAPS is absent (for some low galactic latitude fields), we use the average between USNO B1 and corrected B2. The same was done for the red magnitudes. For an $\mathrm{R}$ magnitude, we take the average between MAPS E and USNO R1/R2, and when MAPS is absent, we use the corrected average USNO R1/R2. The values given in the catalogue are those calculated with the abovementioned principles.

\section{Variability}

The improvement of the USNO B2 and R2 magnitudes allowed us to investigate the sample for variability. We used the average MAPS O/E and USNO B1/R1 measurements for the POSS I observations, and corrected USNO B2/R2 measurements for the POSS II. Some accidental; errors were still present, which we found due to a study of the DSS1/DSS2 fields for possible defects. The variability flags given in the catalogue are based on these data. We did not use SDSS photometry, as the transformation of magnitudes into different bands caused additional errors.

To make a conclusion about the variability, we needed both USNO B and R differences between the two epochs (POSS1 and POSS2) to be large enough and to have nearly the same value (to exclude accidental errors). We took the 3-sigma limit (our measured rms both for B and R) for a confident variability (given as "var"), and 2-sigma limit for probable variability (var:).

Seventy objects matched these criteria; 21 are listed as genuine variables (var) and 49 as probable ones (var:). Of course, there still may be other variables that were not revealed by our approach (which showed approximately the same brightness during both observations). As it is well known, variable objects expected among the FBS BSOs are CVs and some white dwarfs (ZZ Cet stars), as well as a few possible blazars.

\section{SDSS and 2MASS data}

SDSS and 2MASS give accurate astrometric and photometric data for hundreds of million objects, thus being at present the largest and most useful catalogues. We have cross-correlated our sample with accurate positions with 5" search radius with SDSS DR16 and with 2MASS. Though the accuracy of these catalogs is higher, we left the radius larger to avoid accidental errors and losing any association, as a few objects among the FBS BSOs are still expected with larger than $3 \sigma$ errors. In addition, there is a number of extended objects, as well as stars showing proper motion among the FBS BSOs resulting in worse accuracy.

We have found 510 SDSS objects as associations to the FBS BSOs and 970 2MASS objects. The first primary object in SDSS was taken. In addition, 120 SDSS objects have been observed spectroscopically and we were able to classify more FBS objects with unknown nature. SDSS five model magnitudes (ugriz) and 2MASS three NIR magnitudes (JHK) are now available.

\section{SIMBAD and NED associations}

A search for all FBS objects was made in SIMBAD and NED with a 60" search radius around their positions. In SIMBAD, we found 491 objects excluding FBS ones (the FBS objects are also included in SIMBAD and the data given are coming from our previous papers), including 62 extragalactic objects, 4 PN, 414 stars (158 WD, 110 sd, 39 CV, 24 main sequence stars, 83 of unknown type), 7 X-ray sources, and 4 radio sources.

In NED, we have found 196 objects, including 178 optical objects, 10 X-ray, and 8 radio sources. Optical objects include 88 extragalactic ones (including 65 having redshifts), 3 PN, 70 stars, and 17 unknown.

From both SIMBAD and NED objects, some were rejected due to incorrect associations (a neighboring object was taken, which was found from the DSS images), as well as ray and radio sources were combined with associations from non-optical catalogues. The combined SIMBAD/NED results give 522 objects (as 
many objects were the same), including 509 optical ones: 68 extragalactic, 4 PN, $172 \mathrm{WD}, 113$ sd, $38 \mathrm{CV}$, 4 NHB (Normal or Horizontal Branch B stars), 18 other stars, and others are unknown BSOs. Thus, the nature of 414 FBS BSOs is known from other sources and later we have added data from our observations (see subsection "Classification of objects").

\section{Classification of objects and sample content}

All data described in the previous subsections were used to finally classify FBS BSOs, namely data from SIMBAD and NED databases, SDSS DR6, catalogs of AGN, PN, CV, WD (last online updates from Vizier), SDSS white dwarf and CV lists, etc. In addition, individual cross-correlations and DSS image check was carried out for Palomar-Green (PG, Green et al. 1986) objects, which have relatively inaccurate positions and were missed in our previous works. According to the new data, now we have 277 associations in PG, including 20 with very large PG positional errors. 24 more associations are found in Hamburg Quasar Survey (HQS, Hagen et al. 1995).

A spectroscopic study of the FBS BSOs was conducted in 1987-2000 with the Byurakan Astrophysical Observatory 2.6m telescope (BAO-2.6, UAGS and ByuFOSC spectrographs) and Observatoire de HauteProvence 1.93m telescope (OHP-1.93, CARELEC spectrograph). Altogether, 485 slit spectra for 406 objects were obtained, both with photographic (in 1987-1991) and digital (in 1997-2000) receivers. The spectral cov-

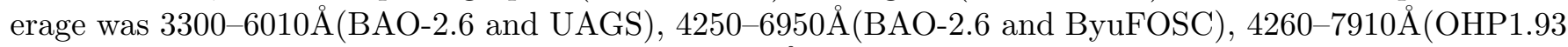
and CARELEC), and the spectral resolution was 6-7Åfor all spectra. Most of the observations were carried out in 1987-1991, however, to have better data, the photographic spectra were digitized and reduced in MIDAS as CCD ones (1850 pixels length). The details are given in Mickaelian et al. (1999, 2001), and Sinamyan \& Mickaelian $(2006,2008 \mathrm{a}, \mathrm{b})$. In Table 1 we give the statistics of spectroscopic observations of the FBS BSOs.

Table 1. Statistics of all spectroscopic observations of FBS blue stellar objects.

\begin{tabular}{|c|c|c|c|c|c|c|c|c|}
\hline \multirow{2}{*}{ FBS zones } & \multicolumn{2}{|c|}{$\begin{array}{c}\text { BAO-2.6 } \\
\mathbf{1 9 8 7 - 1 9 9 1}\end{array}$} & \multicolumn{2}{c|}{$\begin{array}{c}\text { OHP-1.93 } \\
\mathbf{1 9 9 7 - 1 9 9 9}\end{array}$} & \multicolumn{2}{c|}{$\begin{array}{c}\text { BAO-2.6 } \\
\text { 1998-2000 }\end{array}$} & \multicolumn{2}{c|}{ Total $^{*}$} \\
\cline { 2 - 8 } & Objects & Spectra & Objects & Spectra & Objects & Spectra & Objects & Spectra \\
\hline$+\mathbf{3 5}^{\circ}$ & 126 & 139 & 6 & 7 & 0 & 0 & 127 & 146 \\
$\mathbf{+ 3 9}^{\circ}$ & 130 & 151 & 3 & 3 & 1 & 2 & 131 & 156 \\
$\mathbf{+ 4 3}^{\circ}$ & 134 & 158 & 11 & 11 & 2 & 2 & 136 & 171 \\
$\mathbf{+ 6 3}^{\circ} \div+\mathbf{+ 8 6}^{\circ}$ & - & - & 9 & 9 & 3 & 3 & 12 & 12 \\
\hline Total & 390 & 448 & 29 & 30 & 6 & 7 & 406 & 485 \\
\hline
\end{tabular}

* Some objects have been observed using both photographic and digital methods, so the total is different from the sum of the numbers in the individual columns.

As mentioned, altogether 509 objects were known, and 244 more (the new ones only) have been classified from our observations, bringing the total number to 753 (many objects with relatively low-quality spectra have been also re-classified and/or the classification was refined). The classification for WDs and subdwarfs is given as in Green et al. (1986) and McCook \& Sion (2006).

Given that the SDSS spectra have $\sim 1$ Åresolution, for a final classification we have used these spectra when available, and then the other spectra in a decreasing order of resolution.

Thus, at present the physical types of more than $2 / 3$ of our sample are known. These objects are: 217 WDs (DO, DOB, DBA, DAB, DA, DAZ, DZ, and DC subtypes), 371 subdwarfs (sdOA, SDOB, sdOC, sdOD, sdB-O, sdB, sdA, and unknown sd), 26 HBB, 6 NHB, 15 other stars (4 B-A and 11 F-G types, which were probably erroneously included in the sample), $38 \mathrm{CV}, 5$ PNN, 54 AGN (though including 3 HII; all with redshifts), and 14 Galaxies (given as "Gal", 7 with redshifts). According to their spectra, 6 objects are given as "cont" or "cont/Em" (possible AGN or CV), and one object is given as "nonstellar" (possible AGN). Table 2 gives the distribution of FBS BSOs by spectral types. 
Table 2. Distribution of FBS BSO by spectral types.

\begin{tabular}{|c|c|c|c|c|c|c|}
\hline Spectral Type & $\begin{array}{c}\text { Zone } \\
\delta=+35^{\circ}\end{array}$ & $\begin{array}{c}\text { Zone } \\
\delta=+39^{\circ}\end{array}$ & $\begin{array}{c}\text { Zone } \\
\delta=+43^{\circ}\end{array}$ & $\begin{array}{c}\text { Zone } \\
\delta=+63^{\circ} \div+86^{\circ}\end{array}$ & Total & $\mathbf{\%}$ \\
\hline Star* & & 3 & 7 & 5 & $\mathbf{1 5}$ & 2.0 \\
HBB / NHB & 9 & 9 & 12 & 2 & $\mathbf{3 2}$ & 4.2 \\
Sd & 77 & 115 & 119 & 60 & $\mathbf{3 7 1}$ & 49.3 \\
WD & 40 & 41 & 41 & 95 & $\mathbf{2 1 7}$ & 28.8 \\
CV & 8 & 3 & 8 & 19 & $\mathbf{3 8}$ & 5.0 \\
PNN & & 2 & 1 & 2 & $\mathbf{5}$ & 0.7 \\
AGN** & 11 & 10 & 14 & 19 & $\mathbf{5 4}$ & 7.2 \\
Gal & 2 & 5 & 2 & 5 & $\mathbf{1 4}$ & 1.9 \\
cont / nonstellar & 2 & 3 & 1 & 1 & $\mathbf{7}$ & 0.9 \\
\hline Total known & $\mathbf{1 4 9}$ & $\mathbf{1 9 1}$ & $\mathbf{2 0 5}$ & $\mathbf{2 0 8}$ & $\mathbf{7 5 3}$ & $\mathbf{1 0 0 . 0}$ \\
\hline Unknown & 61 & 20 & 28 & 241 & 350 & \\
\hline Total & $\mathbf{2 1 0}$ & $\mathbf{2 1 1}$ & $\mathbf{2 3 3}$ & $\mathbf{4 4 9}$ & $\mathbf{1 1 0 3}$ & \\
\hline
\end{tabular}

* contains the main sequence $A F G$ stars.

** contains QSO, Sy and HII.

\section{The catalogue of the FBS BSOs}

The revised and updated catalogue of the FBS blue stellar objects is given in Mickaelian (2008). The table is available in electronic form at the CDS. The catalogue consists of 1103 objects (the catalog in fact contains 1101 objects, as 2 pairs of objects turned to be identical however we have kept all objects in the list to allow users enter and find objects by all already accepted FBS names). The contents of the FBS catalogue are as follows:

1) First Byurakan Survey (FBS) blue stellar object (BSO) name.

2) FBS BSOs object name $(\mathrm{hhmm}+\mathrm{ddm})$.

3) The original paper number (1-11), where the object was published (see references for FBS lists).

4) FBS zone, where the object was found. Given by the central declination.

5) Comments on object names, including the two pairs of identical objects and alternative FBS names (erroneously used in previous publications).

6) DSS1 J2000 RA (hh:mm:ss.ss), accurate to 1" rms.

7) DSS1 J2000 DEC (dd:mm:ss.s), accurate to 1" rms.

8) DSS2 red J2000 RA (hh:mm:ss.ss), accurate to 1" rms.

9) DSS2 red J2000 DEC (dd:mm:ss.s), accurate to 1" rms.

10) Proper motion (PM) in RA, DEC, total yearly PM, and the position angle of the motion.

11) Galactic coordinates (l and b) (degrees).

12) Object classification into "stars" ("s") and "galaxies" ("g"). This is similar to MAPS classification, which gives an understanding of extension. This is important for magnitude measurements and may lead to large errors in case of misclassification (up to 3-4 magnitudes). Our type is a revised MAPS type based on careful study of the DSS1/DSS2 images.

13) FBS low-dispersion spectral (LDS) type, as explained e.g. in Mickaelian (2000).

14) Comments from inspection of DSS1/DSS2 images (binaries, nearby objects, possible variables, etc.). 
15) Published FBS magnitude (corresponds to B).

16) MAPS O and E magnitudes based on our extension classification (i.e. taken from diameter or integral measurements).

17) USNO-B1.0 B1, R1 (POSS1 epoch), and B2, R2, and I (POSS2 epoch) magnitudes.

18) Summarizing B and R magnitudes derived from MAPS and USNO-B1.0 data.

19) Summarizing B-R color derived from MAPS and USNO-B1.0 data.

20) Variability flags ("var" or "var:").

21) SDSS ugriz model magnitudes from DR6.

22) 2MASS JHK photometry.

23) Other names and optical associations taken from SIMBAD, NED, etc.

24) Final spectroscopic classification based on known data from SIMBAD, NED, etc. and our observations: general type and subtype.

25) Redshifts of the FBS BSOs (extragalactic objects).

26) Associations with X-ray sources.

27) ROSAT X-ray count rate (photons per second).

28) Associations with IR sources (other than 2MASS).

29) IRAS (FSC when available) fluxes at 12, 25, 60, and $100 \mu \mathrm{m}$ (Jansky).

30) Associations with radio sources.

31) Integral $21 \mathrm{~cm}$ flux from NVSS or FIRST (millijansky).

\section{Study of the FBS BSOs sample}

Fig. 1 presents the distribution of the FBS BSOs on the MAPS/USNO-B1.0 O/O-E color-magnitude diagram. Extragalactic objects (AGN and Gal, PNN, CVs, WDs, sd, HBB/NHB, and main sequence stars are marked differently. Objects of each group are in their typical location, however some of the CVs clearly move on the diagram due to their variability hence they may be located anywhere.

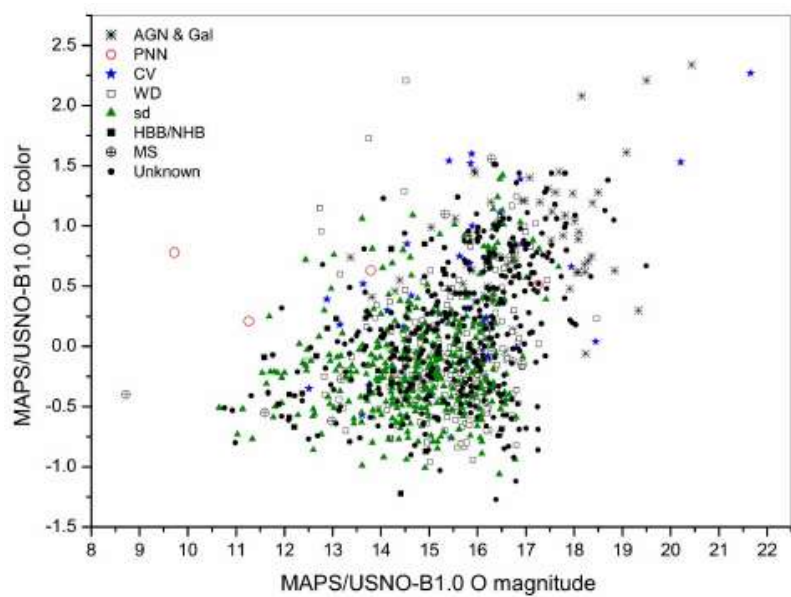

Figure 1. Combined MAPS/USNO-B1.0 O-E vs. $O$ color-magnitude diagram for the FBS blue stellar objects. Asterisks: AGN and Gal; red open circles: PNN; blue stars: CVs; open squares: WDs; green triangles: subdwarfs; filled squares: HBB/NHB; open circles with crosses: main sequence (MS) stars; and filled circles: unclassified objects. 
Fig. 1 shows some biases of the eye selection. Though most of objects show small color indices (O - E $<1.0$ ), however, some selected objects have larger O-E. These are QSOs (selected by their peculiar SEDs), some WDs (selected by their broad absorption lines even without UV excess), and a few other objects.

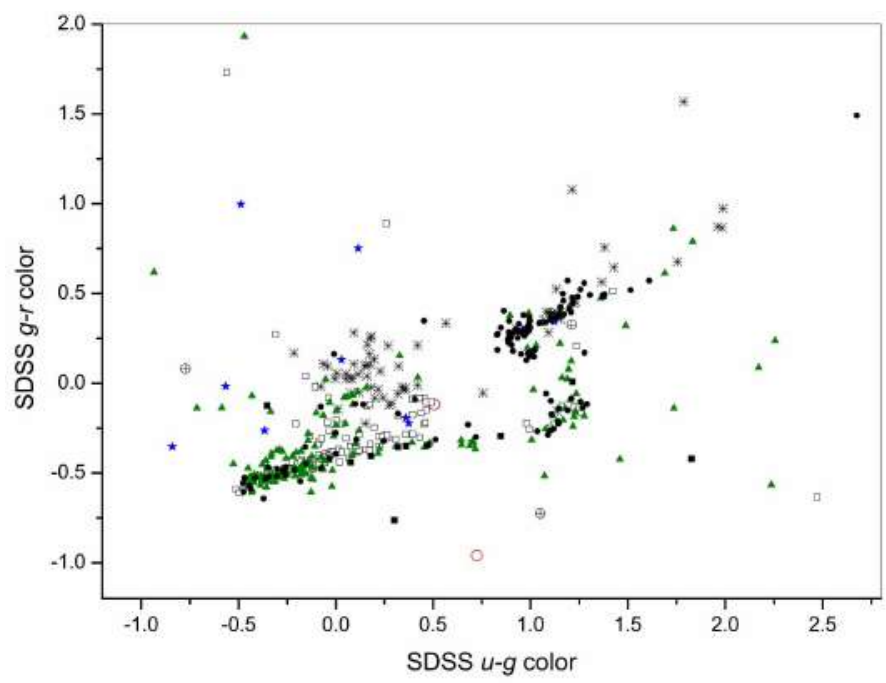

Figure 2. The SDSS $u$ - $g$ vs. $g-r$ color-color diagram for 507 FBS blue stellar objects. The symbols are the same as on Fig. 1

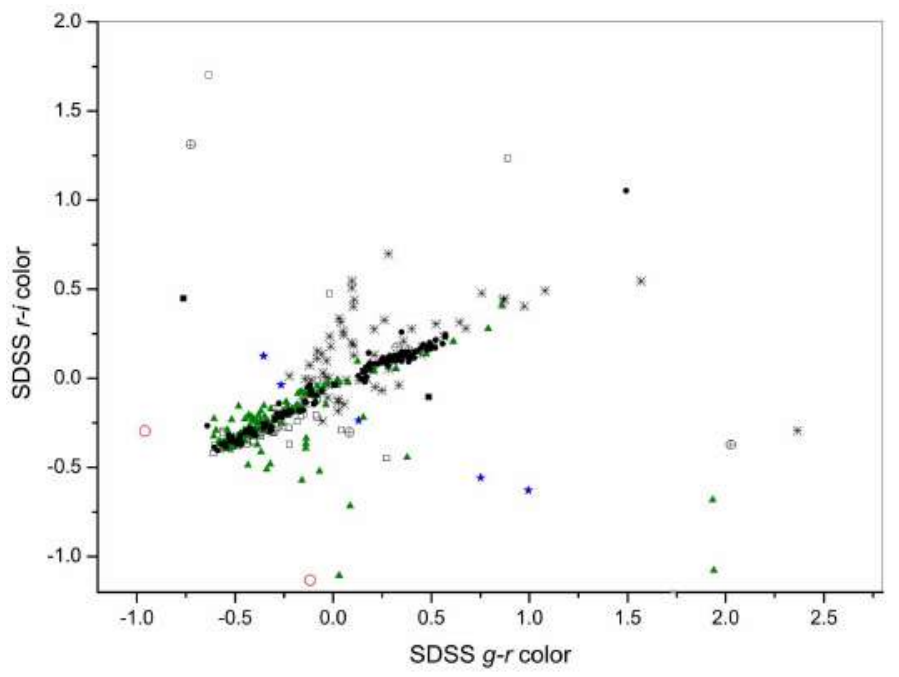

Figure 3. The SDSS $g-r$ vs. $r-i$ color-color diagram for 507 FBS blue stellar objects. The symbols are the same as on Fig. 1

Objects with unknown nature are marked by the filled circles. Their distribution indicates that still many new AGN, CVs, and WD are present in the FBS sample, as well as a few objects with extremely blue colors. Particularly, the faintest objects among the BSOs are either QSOs or CVs at their fainter brightness phase.

There is also a tendency showing that the fainter objects have redder colors. However, this can be also explained by the presence of extragalactic objects in the sample (which are fainter); as QSOs have small $U$ - $B$ color indices but relatively larger $B-V$ ones (which is close to $O$ - $E$ used here). 139 objects (18.5\%) out of 753 classified ones have $O-E>0.50$, which may be considered as relatively non-blue ones. 48 are either extragalactic (AGN and Gal) or show peculiar SEDs (classified as cont or cont/Em:). Three others are PNN, 15 are CVs, 63 are WD/sd/HBBs (including several DZ dwarfs), and 10 are wrongly selected FG stars. If taken objects with $O-E>1.00$, then only 55 objects are left, and 24 are extragalactic ones. Given that the rms accuracy of our derived MAPS/USNO-B1.0 photometric data is $0.4^{m}-0.5^{m}$, we can conclude that the color criterion that corresponds to the selection of FBS BSOs was $O-E<0.50$ and objects having larger color indices should not appear in the catalog and have been selected erroneously.

However, as the FBS BSOs are in fact UVX objects and not necessarily blue ones, we have plotted in 
Fig. 2 and 3 the SDSS (model) $u-g / g-r$ and $g-r / r-i$ color-color diagrams for the 507 FBS objects measured in the SDSS (we have excluded 3 objects with obviously wrong data), including 387 classified and 120 unknown ones. As in Fig. 1, here too different types of objects are marked differently (by the same symbols). The extragalactic objects are much better separated (though some contamination by WDs and subdwarfs is present), which means that one can select new candidate AGN from the unknown FBS objects.

In fact, we do not use the SDSS data as main photometric ones, as it is not for all FBS objects and in addition the photometric accuracy rapidly decreases to the brighter magnitudes, where SDSS objects are saturated.

Fig. 4 gives the 2MASS $J-H / H-K$ diagram for 970 FBS BSOs detected in 2MASS. It seems, 2MASS colors are the best separator for extragalactic objects among the blue stellar ones.

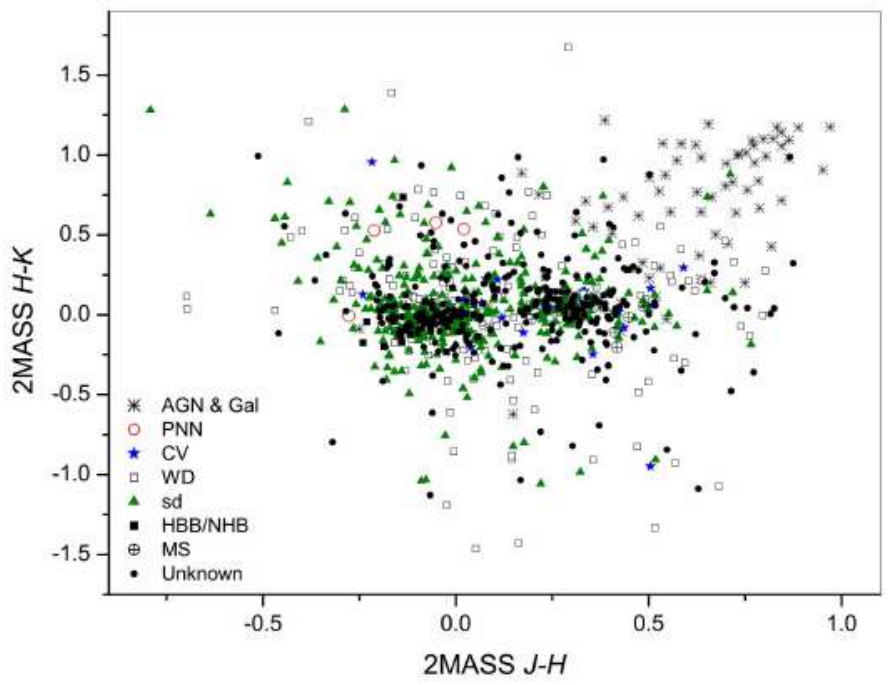

Figure 4. The 2MASS $J-H$ vs. $H$ - $K$ color-color diagram for 970 FBS blue stellar objects. The symbols are the same as on Fig. 1

Based on the analysis of properties of the spectroscopically classified FBS objects and all available data from the multiwavelength catalogs, we have grouped the unknown FBS objects into subsamples of probable AGN (25 new objects are still expected), CVs (20 objects), WDs (101), subdwarfs (173), HBBs (15), and very few other objects.

\section{Summary}

Using newly obtained data on the FBS blue stellar objects, we have revised and updated the FBS sample giving the DSS1/DSS2 (accurate positions and proper motions), MAPS (optical photometry), USNOB1.0 (optical photometry and proper motions), SDSS (ugriz magnitudes) and 2MASS (JHK photometry) catalogues, by re-classifying the objects by means of the slit spectra, and by updating other available data from SIMBAD, NED, X-ray, IR, and radio catalogues. An analysis of the MAPS and USNO-B1.0 photometric data led to a significant improvement of optical magnitudes, which may be used for other studies as well. The available photometric data for FBS BSOs allow us to follow their multiwavelength SEDs and to understand their nature, as not all objects have been observed spectroscopically.

The FBS catalogue contains the main available data for 1101 objects and will be useful as for study of its content so as for studies of individual objects, including AGN, CVs, WDs, etc.

Considering the spectroscopically known objects, our sample contains $7.2 \%$ AGN, $1.9 \%$ other galaxies, $5.0 \%$ CVs, and 28.8\% WDs, slightly more than PG sample $(6.8 \%, 1.9 \%, 3.7 \%$, and $27.0 \%$, respectively). However, this is the reason of more systematic works aimed at discovery of such interesting objects among the BSOs rather than hot subdwarfs, which are mostly the bye-products. Particularly our spectroscopic observations were aimed at discovery of new bright AGN (by pre-selection of candidates from X-ray and radio associations) as well as high proper motion objects (probable WDs), so that most of these objects have probably been already discovered. Hence, the PG and FBS samples are more or less similar by their content.

Most important changes and new details on the FBS BSOs will appear when applying Gaia data; 
proper motions, parallaxes and distances, photometry and variability, and some physical characteristics are expected.

\section{Acknowledgements}

The authors acknowledge support from ANSEF grant in 2021-2022 (PS-astroex-2512) "Discovery and study of new white dwarfs using Gaia accurate astrometry". The Digitized First Byurakan Survey (DFBS) is the digitized version of the Markarian survey (First Byurakan Survey, FBS) plates and is available online at http://www.ia2-byurakan.oats.inaf.it/.

\section{References}

Abrahamian H. V., Mickaelian A. M., 1991, Astrofizika, 35, 197

Abrahamian H. V., Mickaelian A. M., 1993a, Astrofizika, 36, 109

Abrahamian H. V., Mickaelian A. M., 1993b, Astrophysics, 36, 306

Abrahamian H. V., Mickaelian A. M., 1994a, Astrophysics, 37, 27

Abrahamian H. V., Mickaelian A. M., 1994b, Astrophysics, 37, 117

Abrahamian H. V., Mickaelian A. M., 1994c, Astrofizika, 37, 411

Abrahamian H. V., Mickaelian A. M., 1995, Astrofizika, 38, 201

Abrahamian H. V., Mickaelian A. M., 1996, Astrophysics, 39, 315

Abrahamian H. V., Lipovetskyi V. A., Stepanian J. A., 1990a, Astrofizika, 32, 29

Abrahamian H. V., Lipovetski V. A., Mickaelian A. M., Stepanian J. A., 1990b, Astrofizika, 33,345

Abrahamian H. V., Lipovetski V. A., Mickaelian A. M., Stepanian J. A., 1990c, Astrofizika, 33,213

Abrahamian H. V., Lipovetski V. A., Mickaelian A. M., Stepanian J. A., 1991, Astrofizika, 34, 13

Abrahamian H. V., Lipovetski V. A., Mickaelian A. M., Stepanian J. A., 1999, VizieR Online Data Catalog, p. II/223

Ahumada R., et al., 2020, Astrophys. J. Suppl. Ser. , 249, 3

Becker R. H., Helfand D. J., White R. L., Gregg M. D., Laurent-Muehleisen S. A., 2003, VizieR Online Data Catalog, p. VIII/71

Cabanela J. E., Humphreys R. M., Aldering G., Larsen J. A., Odewahn S. C., Thurmes P. M., Cornuelle C. S., 2003, Publ. Astron. Soc. Pac. , 115,837

Condon J. J., Cotton W. D., Greisen E. W., Yin Q. F., Perley R. A., Taylor G. B., Broderick J. J., 1998, Astron. J. , 115, 1693

Cutri R. M., et al., 2003, VizieR Online Data Catalog, p. II/246

Eisenstein D. J., et al., 2006, Astrophys. J. Suppl. Ser. , 167, 40

Eritsyan M. A., Mickaelian A. M., 1993, Astrophysics, 36, 126

Green R. F., Schmidt M., Liebert J., 1986, Astrophys. J. Suppl. Ser. , 61, 305

Hagen H. J., Groote D., Engels D., Reimers D., 1995, Astron. and Astrophys. Suppl. Ser. , 111,195

IRAS 1988, Joint IRAS Science Working Group. Infrared Astronomical Satellite Catalogs, The Point Source Catalog, Version 2.0, NASA RP-1190

Lasker B. M., Doggett J., McLean B., Sturch C., Djorgovski S., de Carvalho R. R., Reid I. N., 1996, in Jacoby G. H., Barnes J., eds, Astronomical Society of the Pacific Conference Series Vol. 101, Astronomical Data Analysis Software and Systems V. p. 88

Markarian B. E., 1967, Astrofizika, 3, 24

Markarian B. E., Lipovetsky V. A., Stepanian J. A., Erastova L. K., Shapovalova A. I., 1989, Soobshcheniya Spetsial'noj Astrofizicheskoj Observatorii, 62,5

Markarian B. E., Lipovetsky V. A., Stepanian J. A., Erastova L. K., Shapovalova A. I., 1997, VizieR Online Data Catalog, p. VII/172

Mazzarella J. M., Balzano V. A., 1986, Astrophys. J. Suppl. Ser. , 62, 751

McCook G. P., Sion E. M., 2006, VizieR Online Data Catalog, p. III/235B

McGlynn T., Scollick K., 1994, in Crabtree D. R., Hanisch R. J., Barnes J., eds, Astronomical Society of the Pacific Conference Series Vol. 61, Astronomical Data Analysis Software and Systems III. p. 34

Mickaelian A., 1994, PhD thesis, Byurakan Astrophysical Observatory (BAO)

Mickaelian A. M., 2000, Astronomical and Astrophysical Transactions, 18, 557 
Mickaelian A. M., 2004, Astron. Astrophys. , 426, 367

Mickaelian A. M., 2005, in Sion E. M., Vennes S., Shipman H. L., eds, Astrophysics and Space Science Library Vol. 332, White dwarfs: cosmological and galactic probes. pp 61-72, doi:10.1007/1-4020-3725-2_7

Mickaelian A. M., 2008, Astron. J. , 136, 946

Mickaelian A. M., Gigoyan K. S., 2006, Astron. Astrophys. , 455, 765

Mickaelian A. M., Sinamyan P. K., 2010, Mon. Not. R. Astron. Soc. , 407, 681

Mickaelian A. M., Eritsyan M. A., Abramyan G. V., 1991, Astrophysics, 34, 186

Mickaelian A. M., Gonçales A. C., Véron-Cetty M. P., Véron P., 1999, Astrophysics, 42, 1

Mickaelian A. M., Gonçalves A. C., Véron-Cetty M. P., Véron P., 2001, Astrophysics, 44, 14

Mickaelian A. M., Abrahamian H. V., Guibert J., Chesnel R., 2002a, Astrophysics, 45, 73

Mickaelian A. M., Balayan S. K., Ilovaisky S. A., Chevalier C., Véron-Cetty M. P., Véron P., 2002b, Astron. Astrophys. , 381,894

Mickaelian A. M., et al., 2007, Astron. Astrophys. , 464, 1177

Mickaelian A. M., Mikayelyan G. A., Sinamyan P. K., 2011, Mon. Not. R. Astron. Soc. , 415, 1061

Monet D. G., et al., 2003, Astron. J. , 125, 984

Moshir M., et al. 1990, IRAS Faint Source Catalogue, p. 0

Nesci R., Mickaelian A., Rossi C., 2009, The Astronomer's Telegram, 2338, 1

Ritter H., Kolb U., 2003, Astron. Astrophys. , 404, 301

Schmidt M., Green R. F., 1983, Astrophys. J. , 269, 352

Silvestri N. M., et al., 2006, Astron. J. , 131, 1674

Sinamyan P. K., Mickaelian A. M., 2006, Astrophysics, 49, 333

Sinamyan P. K., Mickaelian A. M., 2008a, Astrophysics, 51, 37

Sinamyan P. K., Mickaelian A. M., 2008b, Astrophysics, 51, 226

Sinamyan P. K., Mickaelian A. M., 2009, Astrophysics, 52, 76

Sinamyan P. K., Mickaelian A. M., 2011, Astrophysics, 54, 403

Szkody P., et al., 2007, Astron. J. , 134, 185

Véron-Cetty M. P., Véron P., 2006, Astron. Astrophys. , 455, 773

Voges W., et al., 1999, Astron. Astrophys. , 349, 389

Voges W., et al., 2000, MPE, Garching, 7432, 3

Weedman D. W., 1977, Vistas in Astronomy, 21, 55

Weedman D. W., Khachikyan E. E., 1968, Astrophysics, 4, 243 